\title{
Phase Unwrapping based on the Modified Discrete Cosine Transform and the Branch Method
}

\author{
Chen Xingtong \\ College of Mining Engineering \\ North China University of Science and Technology \\ Tangshan, China
}

\begin{abstract}
InSAR technology belongs to microwave remote sensing technology. Because of the wide application of InSAR technology, it is gradually popularized. Because of its high data processing and higher precision, phase unwrapping directly affect the final digital elevation information. The phase unwrapping process in InSAR data processing has a great influence on the accuracy of the DEM, so it is a key step to improve the accuracy of phase unwrapping. Through the research and analysis of direction cosine and the branch method. This paper improves the results of phase unwrapping by combining the modified discrete cosine transform and the branch method. This experiment shows that the combining model can complement each other, which would be a better solution to filter out the speckle noise and maintain the image detail. It further proves the significance of InSAR technology and its great advantage and potential.
\end{abstract}

Keywords-InSAR; Phase Unwrapping; Branch Method; Direction Cosine ; Combining Method

\section{INTRODUCTION}

The branch cut algorithm not only is the simplest algorithm, but also the fastest algorithm. But due to ignore the quality problem of the phase values, this may lead to split path generated by position errors. The algorithm is easy to form "isolated island", which leads to the failure of solution [1-2]. The DCT algorithm can effectively solve the interference image to achieve the optimal results, but cannot identify the singular point and cause the 2 of transmission. Therefore, this paper chooses the DCT algorithm and the method of branch tangent to solve the problem of phase unwrapping.

In Twentieth Century, the one-dimensional integral thought is extended to the two-dimensional phase unwrapping [3]. In the ideal state, there is no noise interference in the process of phase unwrapping, so the two dimensional phase unwrapping is simplified as a method of solving integral. However, the phase unwrapping process may have the interference of noise [45]. There are a certain number of singular points. These singular points may cause error propagation, so it is important to avoid these singular points and ensure the accuracy of results.

\author{
Cai Yang
College of Mining Engineering \\ Cai Yang
College of Mining Engineering \\ North China University of Science and Technology \\ Tangshan, China \\ E-mail: 550910904@qq.com
$*$ Corresponding Author \\ E-mail: 550910904@qq.com
* Corresponding Author
}

\section{THE MODIFIED DISCRETE COSINE TRANSFORM METHOD}

\section{A. The Theory of Discrete Cosine Transform Method}

The transformation process of discrete cosine transform (DCT) method is as follows [6]:

First step: forward 2D DCT transform

$$
\begin{gathered}
C_{m, n}\left\{\sum_{i=1}^{M} \sum_{j=1}^{N} 4 X_{i, j} \cos \left[\frac{\pi}{2 M} m(2 i+1)\right] \cos \left[\frac{\pi}{2 N} n(2 j+1)\right]\right. \\
1 \leq i \leq M, 1 \leq j \leq N
\end{gathered}
$$

Second step: inverse DCT transform

$$
\begin{gathered}
X_{i, j}=\left\{\frac{1}{M N} \sum_{m=1}^{M} \sum_{n=1}^{N} \omega_{1}(m) \omega_{2}(m) \cos \left[\frac{\pi}{2 M} m(2 i+1)\right] \cos \left[\frac{\pi}{2 N} n(2 i+1)\right]\right. \\
1 \leq i \leq M, 1 \leq j \leq N \\
m=1 \omega_{1}(m)=\frac{1}{2} ; 2 \leq m \leq M \quad \omega_{1}(m)=1
\end{gathered}
$$

The third step: the use of the automatic boundary conditions

$$
\phi(i, j)=\frac{p(i, j)}{2\left(\cos \frac{\pi i}{M}+\cos \frac{\pi j}{N}-2\right)}
$$

$M, N$ represent the ranks of the image respectively

$$
p(i, j)=\Delta_{x}(i, j)-\Delta_{x}(i-1, j)+\Delta_{y}(i, j)-\Delta_{y}(i, j-1)
$$


The final phase unwrapping phase can be obtained by inverse transforming the above equation [7].

Among them, $\phi_{i, j}$ is the phase value of the

\section{B. The Improved DCT Algorithm}

The traditional DCT transform algorithm is based on two-way different processing, mainly on the horizontal and vertical difference, and improved DCT is based on four directions of the classified information extraction phase unwrapping information. That is horizontal, vertical, left and right diagonal.

$$
\begin{gathered}
\Delta_{i, j}^{l}=\omega\left[\phi_{i+1, j-1}-\phi_{i, j}\right] \\
\Delta_{i, j}^{r}=\omega\left[\phi_{i+1, j+1}-\phi_{i, j}\right]
\end{gathered}
$$

$\phi_{i, j}$ can be derived in the DCT improved algorithm:

$p_{i, j}=\left(\phi_{i+1, j}-2 \phi_{i, j}+\phi_{i-1, j}\right)+\left(\phi_{i, j+1}-2 \phi_{i, j}-\phi_{i, j-1}\right)$ $+\left(\phi_{i+1, j+1}-2 \phi_{i, j}+\phi_{i-1, j-1}\right)+\left(\phi_{i+1, j-1}-2 \phi_{i, j}+\phi_{i-1, j+1}\right)$

\section{Combining DCT Algorithm And Branch Tangent Method}

Branch cut method is a powerful noise-immune algorithm for correct phase unwrapping of noisy phase maps. The shortest branch cut length promises the optimal unwrapping result of the wrapped phase maps. The nearest rules to connect residue is to form a branch cut line and isolate the noise [8-9]. Restoring the absolute phase, by identifying the singular point, preventing the error transfer and winding phase gradient in the form of integral adjacent pixels.

Residual always follow the principle is from top to bottom, from left to right [10]. After taking the first residual point as the center, searching the second residual with $3^{*} 3$. If there is no point in the search, increasing the search range searching the area with $5^{*} 5$, the two residuals are connected by the branch tangent to the charge balance.

The mathematical model of Branch-cut is as follows:

First: calculate the main phase of winding value

$$
\begin{gathered}
\Delta_{1}=\omega\{\phi(i, j+1)-\phi(i, j)\} \\
\Delta_{2}=\omega\{\phi(i+1, j+1)-\phi(i, j+1)\} \\
\Delta_{3}=\omega\{\phi(i+1, j+1)-\phi(i, j+1)\} \\
\Delta_{4}=\omega\{\phi(i, j)-\phi(i+1, j)\}
\end{gathered}
$$

Second: calculate phase gradient value of minimum closed path

$$
\text { sum }=\Delta_{1}+\Delta_{2}+\Delta_{3}+\Delta_{4}=0
$$

The synthesis algorithm is to use the branch tangent method to recognize the singular points, and then use DCT algorithm to deal with the difference.

\section{APPLICATION EXAMPLE}

The experiment mainly uses the PALSAR data of ALOS satellite, the selected study area is $110.04 \mathrm{oE} \sim 110.09 \mathrm{oE}$, the latitude range is $39.260 \mathrm{~N} \sim 39.31 \mathrm{oN}$. This field covers an area of 2.67 square kilometers, with recoverable reserves of 1941, 0000 tons of mine. The Coal mine was constructed in 1987 and put into operation in December, 1990. It is the only coal open-pit coal mine. After coal mining, the surface subsidence, destroy the natural environment for human survival and development. The ground subsidence monitoring is an important part of coal mining and can continue to develop. InSAR technique is used to carry out the settlement monitoring, and the phase unwrapping is the key point in this paper.

The example uses the PALSAR data of ALOS satellite to monitor the surface subsidence by $\mathrm{L}$ band. HHALPSRP252290780, HHALPSRP24558078055 are the date names.

Before the phase unwrapping, the baseline estimation must be carried out to generate the interference pattern. The baseline is a necessary parameter for the inversion of the ground point elevation and the surface deformation.

TABLE I. BASELINE DATA

\begin{tabular}{cccc}
\hline & InSAR image & $\begin{array}{c}\text { Vertical } \\
\text { distance }\end{array}$ & $\begin{array}{c}\text { Critical } \\
\text { Baseline }\end{array}$ \\
\hline HHALPSRP & HHALPSRP & 213.464 & $|7001.394|$ \\
252290780 & 2455807804 & & \\
HHALPSRP & HHALPSRP & 715.719 & $|7001.394|$ \\
252290780 & 24558078055 & & \\
\hline
\end{tabular}

Through theoretical study of InSAR technology, the requirements of radar image are higher, which is mainly to consider the influence of baseline. A large number of previous experiments conclude that the vertical baseline distance is less than $1 / 3$ of the critical baseline. When the baseline is increased to a certain extent, the image coherence will be reduced to 0 .The critical value is called critical baseline.

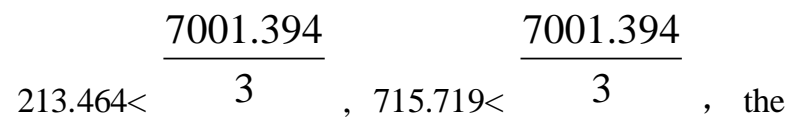
results of the baseline estimate are in line with the requirements.

After baseline estimation, the interference pattern is generated. Interference graph not only contains real part and imaginary part but also contains the main auxiliary phase difference of the image. The phase difference may also deformation phase components, the atmosphere caused by the phase component. The Fig.1 is the interference map. 


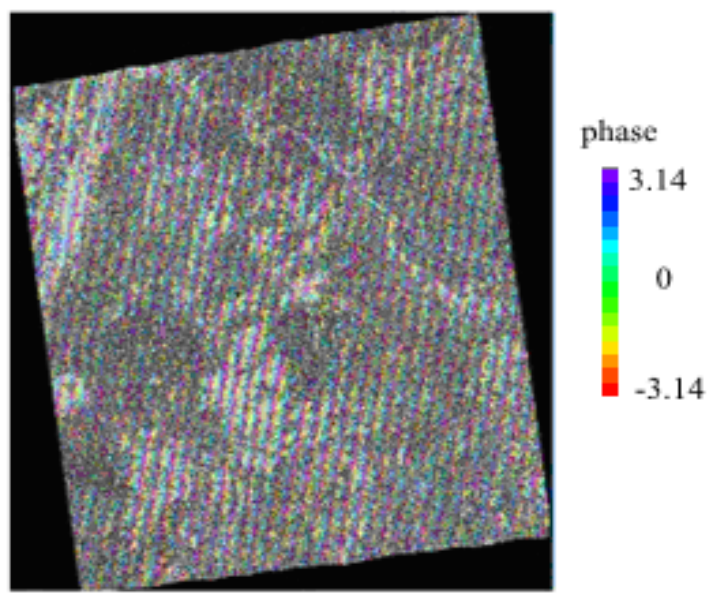

Figure 1. Image of Interference

Because of the existence of the ground effect and noise the interference fringes are dense and the terrain changes are covered. The interference in the weak noise region is shown as a narrow band signal, and the signal of the interference in the region of the noise is shown as a broadband signal,

There is a lot of noise after flat earth effect. The noise will lead to phase discontinuous and inconsistent, so need for filter the interferogram. I, Goldstein filtering method is used in this paper.

The Fig. 2 is the interference pattern after flatting and the filtering.

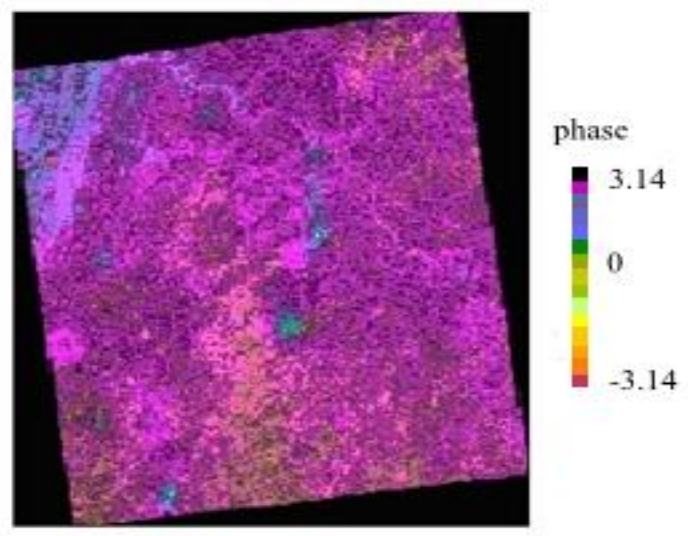

Figure 2. Image of Filtering Interference

From the interferogram, the upper left corner has a large noise, and also has certain singular points, and this is difficult to unwrapping.

Using the following circumstances, memory: $2 \mathrm{G}$; operating system: Windows XP; software VC6.0 to phase unwrapping. The results are as follows:

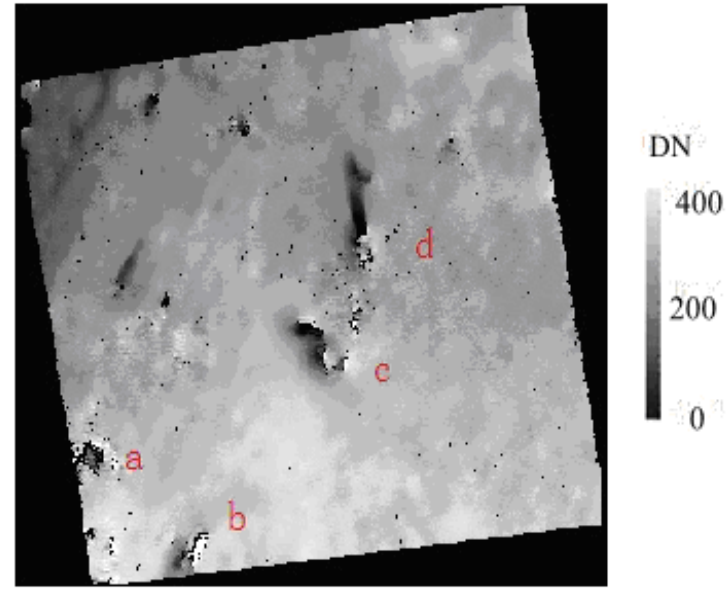

Figure 3. Image of Unwrapped Phase Using Combining Algorithm

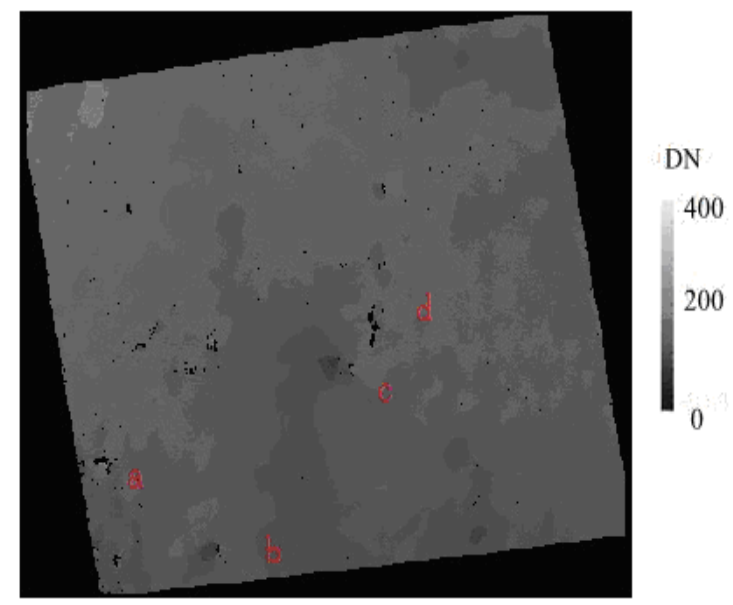

Figure 4. Image of Unwrapped Phase Using Improved DCT Algorithm

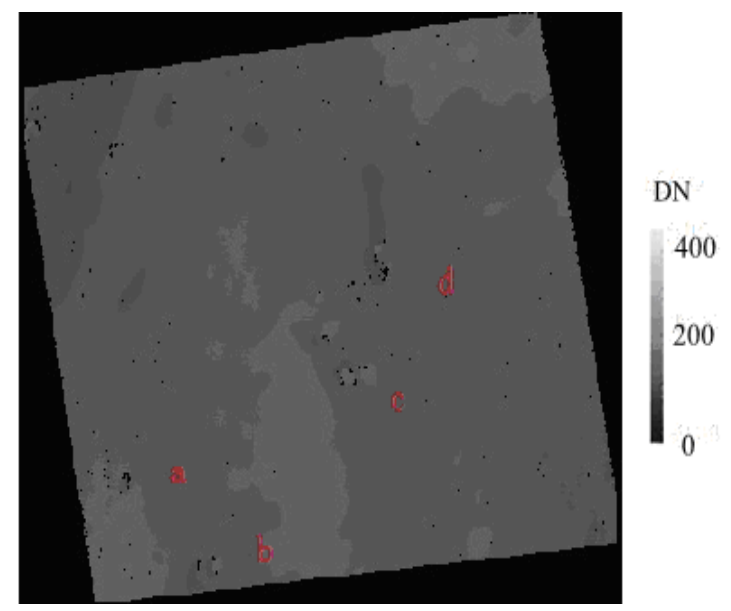

Figure 5. Image of Unwrapped Phase Using DCT Algorithm

Through Fig.2, Fig. 3 and Fig. 4, the area marked with a, $\mathrm{b}, \mathrm{c}$ and $\mathrm{d}$, the topography of image 2 is obvious. That is, the solution is best.

The improved algorithm proposed in this paper effectively avoids the error transfer caused by the singular point, and the unwrapping effect is improved obviously. 
The following is a comparison of three algorithms from quantitative analysis.

TABLE II. A COMPARISON OF THREE ALGORITHMS

\begin{tabular}{ccc}
\hline method & time & winding value \\
\hline DCT algorithm & $2.948664 \mathrm{~s}$ & 0.5632 \\
Improved DCT algorithm & $3.586827 \mathrm{~s}$ & -0.0632 \\
combining algorithm & $4.376438 \mathrm{~s}$ & -0.0256 \\
\hline
\end{tabular}

1). Although DCT operation time is the shortest, its solution is the worst.

2). The improved DCT algorithm makes up for the shortcomings of DCT algorithm, and improves understanding bound precision, but also increases time.

3). Compared to the above two algorithms, the combination of improved DCT and branch tangent algorithm are the best, but the time is also the longest.

\section{CONCLUSION}

From the quantitative analysis, it can be seen that the combination of the improved DCT and the branch tangent method takes a long time, but the effect is the best. Especially in the region of large noise and singular point, it is effective to avoid the error caused by passing through a singular point, which proves the validity of this method.

\section{REFERENCES}

[1] Matias R. Viotti, Analucia V. Fantin, Armando Albertazzi, Daniel P. Willemann. Processing of noised residual stress phase maps by using a 3D phase unwrapping algorithm $[\mathrm{J}]$. Optics and Lasers in Engineering, 2013:

[2] Limei Song,Xiaoxiao Dong,Jiangtao Xi,Yanguang Yu, Chaokui Yang. A new phase unwrapping algorithm based on Three Wavelength Phase Shift Profilometry method [J]. Optics and Laser Technology, 2013, 45:

[3] Yuan Guo,Xiaotian Chen,Tao Zhang. Robust phase unwrapping algorithm based on least squares [J]. Optics and Lasers in Engineering, 2014, 63:

[4] Zhang Li, Liao Ming Sheng, Zhang Zuxun, Zhang Yong. The coherence coefficient map and residue guided least square phase unwrapping algorithm [J]. Geo-spatial Information Science,1999, 21:.

[5] Feipeng Da,Hao Huang. A fast, accurate phase unwrapping method for wavelet-transform profilometry $[\mathrm{J}]$. Optics Communications, 2011, 2854:

[6] Julio C. Estrada, M. Servin,Javier Vargas. 2D simultaneous phase unwrapping and filtering: A review and comparison $[\mathrm{J}]$. Optics and Lasers in Engineering, 2012, 508:

[7] Xianyu Su,Wenjing Chen. Reliability-guided phase unwrapping algorithm: a review [J]. Optics and Lasers in Engineering, 2003, 423:

[8] Wei Li,Alexandru V. Avram,Bing Wu,Xue Xiao,Chunlei Liu. Integrated Laplacian - based phase unwrapping and background phase removal for quantitative susceptibility mapping $[\mathrm{J}]$. NMR Biomed., 2014, 272:

[9] Samia Heshmat, Satoshi Tomioka, Shusuke Nishiyama. Performance Evaluation of Phase Unwrapping Algorithms for Noisy Phase Measurements [J]. International Journal of Optomechatronics, 2014, 84:

[10] A.H. Kattoush. An N-Dimensional Recurrent Noisy Phase Unwrapping Algorithm [J]. International Journal of Computers and Applications,

294:. 